\title{
Wie sag ich's der Bevölkerung? Evaluation einer Ausstellung und eines Lehrpfads zum Thema «Alpine Naturgefahren»
}

\author{
Klaus Wagner, Freising
}

\section{Einleitung}

«In vielen Fällen werden mit den Instrumenten der Bauvorsorge und der Verhaltensvorsorge größere Schadensminderungsquoten zu erreichen sein, als über alle Maßnahmen des natürlichen Wasserrückhalts und des technischen Hochwasserschutzes zusammen. Voraussetzung dafür ist jedoch, da $\beta$ dem Einzelnen auch sein Teil der Verantwortung bei der Hochwasservorsorge bewußt gemacht wird" (LÄNDERARBEITSGEMEINSCHAFT HOCHWASSER 1995: 16).

Diese Aussage verdeutlicht die Vorstellung in Verwaltung und Politik, dass aufgrund der Beschränktheit von technischen Maßnahmen der Flächen-, Bau-, Risikound Verhaltensvorsorge ein zunehmender Stellenwert beizumessen ist. Die Länderarbeitsgemeinschaft Hochwasser hält in diesem Zusammenhang einen Bewusstseinswandel in der Bevölkerung für notwendig. Wie aber dieser Bewusstseinswandel initiiert werden soll, ist unklar, da die Wirkungen von Aufklärungskampagnen, Informationsbroschüren und Faltblättern über Naturgefahren weitgehend unbekannt sind. Wie Sims \& BAUMANN (1983: 167) darstellten, muss von dem «education causes awareness causes behavior'-Mythos aufgrund vielfacher empirischer Belege Abschied genommen werden. Vielmehr gilt:

"Sometimes, under highly specified conditions, and if properly executed, with certain target publics, information may lead to awareness and awareness may lead to behavior»(ebd.: 167).

Die Wirkung von Aufklärungskampagnen in anderen Themenbereichen wurde von vielen Forschungsrichtungen analysiert. Nach BECKER \& KistLer (1992: 80) sind vor allem Ansätze der Kommunikations- bzw. Medienwirkungsforschung, der Werbewirkungs- und der Persuasionsforschung interessant. Übereinstimmend gehen alle neueren Ansätze davon aus, dass die Wirkung von Informationen nicht alleine durch die Botschaften, sondern vor allem durch das Rezipientenverhalten und dessen Einstellungen, Wissen und Überzeugungen geprägt wird.

Die Wirkung von Informationskampagnen über alpine Naturgefahren wurde bisher nicht wissenschaftlich analysiert. Im Bereich der Naturgefahren wurden die meisten Studien in den USA zur Wirkung der Aufklärung über Erdbeben (vgl. Lindell \& Perry 2000) erstellt.
Aufgrund fehlender Kenntnisse über die Wirkung von Informationsinstrumenten über alpine Naturgefahren wurde im Rahmen des Projekts «Naturgefahrenbewusstsein und -kommunikation im Bayerischen Alpenraum» diesem Thema ein hoher Stellenwert eingeräumt. In drei Untersuchungsgemeinden (Benediktbeuern, Hindelang und Tegernsee) wurde mittels qualitativer und quantitativer Befragungen das Naturgefahrenbewusstsein erhoben. In Zusammenarbeit mit den Gemeinden und den Wasserwirtschaftsämtern wurden Kommunikationswege und -mittel entwickelt und erprobt. Bei der Evaluation wurde den Fragen nachgegangen, wie die Informationen wahrgenommen und verarbeitet wurden sowie welche Wissens- und Einstellungsänderungen bei den Rezipienten beobachtet werden können. Im Folgenden steht die Evaluation einer Ausstellung und eines Lehrpfads im Vordergrund, da anhand dieser Beispiele der Informationsverarbeitungsprozess sehr gut beschrieben werden kann.

\section{Theoretische Anmerkungen}

In dem diesem Projekt zugrunde liegenden Modell von Merten (1994: 311-312) wird

"die Wirkung von Kommunikation an drei Bündeln von Wirkfaktoren festgemacht, nämlich 1) am Informationsangebot (dem Stimulus), 2) dem internen Kontext, der durch Erfahrungen. Wissen und Einstellungen des Rezipienten, aber auch durch seine situative Disposition bestimmt ist und 3) durch den externen Kontext, der vor allem durch situative und soziale Randbedingungen (z.B. Anwesenheit anderer bei der Rezeption, Normen. Werte, Medienverfassung) definiert ist.»

Der Rezipient wählt also aus dem gegebenen Informationsangebot entsprechend seines internen und externen Kontexts bestimmte Informationen aus. Die Verarbeitung dieser Informationen geschieht wiederum vor dem Hintergrund des internen Kontexts. Wie Deutsch (1973:215) erläutert, werden vor allem die Informationen wahrgenommen bzw. verarbeitet, die zu dem eigenen Wissensstand anknüpfungsfähig sind. Aufgrund der niedrigen Bedeutung des Naturgefahrenthemas für das alltägliche Leben (GEIPEL et al. 1997: 28; SimS \& BAUMANN 1983: 183) wird erwartet, dass andere Wissensbereiche und Einstellungen die Wahrnehmung alpiner Naturgefahren beeinflussen. Sie wirken also wie Brillen, durch die Informationen über Naturgefahren scharf oder verzerrt wahrgenommen werden. Der Begriff der Brille wurde durch WeichSELGaRTNER 
(2001) beeinflusst, der in seiner Dissertation die unterschiedlichen Sichtweisen der Wissenschaftsdisziplinen auf die Naturgefahrenthematik beschreibt. Mit dem griffigen Bild der Brille soll ein Begriff eingeführt werden, der auch für den Praktiker spontan eingängig ist, um so die «irrationale» Sicht der Laien besser verständlich zu machen.

\section{Material und Methoden}

\subsection{Grundsätzliche Überlegungen}

Die Ausstellung und der Lehrpfad, die im Folgenden genauer beschrieben werden, sind aufgrund der situativen Rahmenbedingungen und ihres ähnlichen inhaltlichen Konzepts vergleichbar. In beiden Fällen entschieden sich die meisten Personen spontan, sich trotz ihres Ziels, wandern zu gehen bzw. in Rosenheim einzukaufen, mit der Ausstellung bzw. dem Lehrpfad zu beschäftigen. Sowohl in der Ausstellung als auch beim Lehrpfad stand das Thema «Schutz vor alpinen Naturgefahren» im Vordergrund, wobei aber das Thema «Ökologie der Wildbäche» nicht ausgespart wurde.

In beiden Fällen wurden die Besucher sowohl beobachtet als auch nach Verlassen der Ausstellung bzw. des Bereichs des Lehrpfads befragt. Die Beobachtung diente dazu, die selektive Wahrnehmung des Informationsangebots zu erfassen. Um die Ergebnisse der Beobachtung zwischen den unterschiedlichen Ausstellungsteilen und dem Lehrpfad vergleichen zu können, wurde eine Norm-Lesezeit für jede Informationstafel ermittelt. Die Norm-Lesezeit ist die Zeit, die ein Betrachter zum Lesen der gesamten Tafel und zum Erfassen der Bilder und Graphiken braucht. Sie stellt somit eine Untergrenze dafür dar, um den gesamten Inhalt einer Tafel aufzunehmen. Diese Zeit wurde von jeweils zwei unabhängigen Versuchspersonen mittels Stoppuhr erhoben.

Die Wirkung der Ausstellung und des Lehrpfads auf den internen Kontext der Besucher, d.h. deren Wissen und Einstellungen bezüglich alpiner Naturgefahren, wurde durch den Vergleich von Personen, die sich intensiv mit dem Dargebotenen beschäftigt hatten, mit solchen, die sich nur oberflächlich bzw. nicht damit beschäftigt hatten, ermittelt. Aufgrund des Fehlens einer Vorbefragung konnten die Wissensunterschiede nicht direkt ermittelt, sondern nur durch den beschriebenen Vergleich der Gruppen abgeleitet werden. Auf eine Vorbefragung wurde verzichtet, da dadurch das Verhalten der Besucher beeinflusst worden wäre. Für die Vor- und Nachteile des Versuchsdesigns siehe Schnell et al. (1995: $214 \mathrm{ff}$.) oder spezifisch für die Evaluation von Risikokommunikationsstrategien Rohrmann (2000: 212 f.).

\subsection{Ausstellung «100 Jahre Wildbachverbauung»}

Die Ausstellung wurde zum 100-jährigen Bestehen der Bayerischen Wasserwirtschaftsverwaltung in einem Stadtpark Rosenheims errichtet. Die Ausstellung war im Zeitraum 10.7.02-1.9.02 täglich geöffnet. Der Eintritt war frei. Die Ausstellung bestand aus einem in Blockhausbauweise erstellten Ausstellungsgebäude und einem Freiluftgelände. Vom Dach des Ausstellungsgebäudes entsprang ein Wildbach, der mit Verbauungsmaßnahmen im Modellmaßstab das Thema der Ausstellung veranschaulichte. Im Inneren des Blockhauses wurden überwiegend mittels Ausstellungstafeln die Themen Schutz vor Gefahren durch Wildbäche und Massenbewegungen, Schutzwaldsanierung, Ökologie der Wildbäche und Fischerei vermittelt. Ein großer Anziehungspunkt war ein Tunnelaquarium in der Mitte des Ausstellungsgebäudes.

\subsection{Lehrpfad am Lainbach}

Der Lehrpfad wurde 1996 vom Wasserwirtschaftsamt Weilheim erstellt. Auf einer Länge von $4 \mathrm{~km}$ werden ca. 150 Höhenmeter bewältigt. Zwei Übersichtstafeln und zehn thematische Tafeln weisen die Besucher auf die Themen Entstehungsprozesse von Wildbachgefahren, Schutzmaßnahmen, Naturraum/Ökologie und die Aufgaben der Wasserwirtschaftsverwaltung hin. Die Informationstafeln sind aus Bildern, die durch kurze Erklärungen bzw. auch längere Textabschnitte erläutert werden, aufgebaut.

\subsection{Beschreibung der Erhebungsinstrumente}

Die standardisierten Befragungen beschäftigten sich mit den Themen

- Auslöser und Intensität des Ausstellungs- bzw. Lehrpfadbesuchs (geschlossene Fragen)

- Bewertung und Wirkungen der Ausstellung bzw. des Lehrpfads (meist offene Fragen mit Feldkodierung)

- Soziodemographische Variablen.

Die verwendete Fachbegriffe sowie die bekannten Schwierigkeiten der Erhebungsinstrumente werden hier nicht erläutert. Dies kann der entsprechenden Fachliteratur entnommen werden (DIEKMANN 2001; SChNell et al. 1995; Friedrichs 1985). Die Verweigerungsquote betrug bei der Befragung am Lainbach weniger als $20 \%$, bei der Ausstellung $47 \%$. Dies lag daran, dass Personen, die nur kurz die Ausstellung besuchten, nicht bereit waren, sich 15-30 Minuten für die Befragung Zeit zu nehmen.

Das Beobachtungskonzept unterschied sich deutlich zwischen den Erhebungen. In Rosenheim wurde jeweils eine Person verdeckt während des gesamten Ausstellungsbesuchs beobachtet. Dabei wurden der Besuchsweg, die Betrachtungs- und Verweilzeiten an den Ausstellungstafeln und -objekten aufgenommen. Wegen der langen Gehzeiten zwischen den Tafeln des Lehrpfads wurden die Beobachter am Lainbach jeweils in der 


\begin{tabular}{|c|c|c|}
\hline & Ausstellung in Rosenheim & Lehrpfad am Lainbach \\
\hline \multicolumn{3}{|c|}{ Beobachtung } \\
\hline Anzahl/Geschlecht & 109 Personen (48\% Frauen) & 297 Personen (48\% Frauen) \\
\hline \multicolumn{3}{|c|}{ Befragung } \\
\hline $\begin{array}{l}\text { Anzahl/Geschlecht } \\
\text { Alter (Median) } \\
\text { Schulabschluss }\end{array}$ & $\begin{array}{l}\text { 95 Personen (40\% Frauen) } \\
\text { 11-74 Jahre (44 Jahre) } \\
\text { Haupt-/Realschule: } \\
\text { (Fach-)Abitur: }\end{array}$ & $\begin{array}{l}\text { 74 Personen (33\% Frauen)* } \\
\text { 15-84 Jahre (44 Jahre) } \\
\text { Haupt-/Realschule: } \\
\text { (Fach-)Abitur: }\end{array}$ \\
\hline
\end{tabular}

* Teilweise beteiligte sich die gesamte Wandergruppe an der Befragung.

Tab. 1: Übersicht über die Untersuchungskollektive Sample characteristics

Aperçu des collectifs d'étude
Nähe der Lehrpfadtafel postiert. Aufgrund der begrenzten Anzahl der Beobachter wurden nur vier der zehn thematischen Tafeln beobachtet. Um die Ergebnisse der einzelnen Beobachtungspunkte und der Befragung miteinander verknüpfen zu können, wurden für jede Person unveränderliche Merkmale wie Geschlecht, Haarfarbe und -länge sowie Farbe der Kleidung notiert.

\subsection{Untersuchungskollektive}

Wie Tab. 1 zu entnehmen ist, besuchten ungefähr gleich viele Männer wie Frauen die Ausstellung sowie den Lehrpfad. Bei den Befragungen überwog der Anteil der Männer, da bei gemischt geschlechtlichen Gruppen eher die Männer an der Befragung teilnahmen. Diese Selbstselektion konnte durch die Befrager nicht verhindert werden. Bei der Befragung in Rosenheim gab es aufgrund der hohen Verweigerungsquote einen weiteren systematischen Unterschied zwischen dem Befragten- und dem Beobachtungskollektiv. An der Befragung beteiligten sich überwiegend Personen, die ohne Kinder die Ausstellung besuchten bzw. länger als der Durchschnittsbesucher in der Ausstellung blieben.
Die Unterschiede zwischen den Befragungskollektiven der beiden Untersuchungen waren hinsichtlich des Alters, des Geschlechts und des Bildungsniveaus nur gering.

\section{Ergebnisse}

\subsection{Selektive Wahrnehmung}

Beim Lehrpfad am Lainbach wurden die einzelnen Tafeln durchschnittlich von $27 \%$ der Passanten betrachtet. Überdurchschnittlich hoch war das Interesse bei den Tafeln «Lebensraum Wildbach: Pflanzen» und «Treibholzrechen» (siehe Tab. 2). Am ehesten wurden die Tafeln beim Bergaufgehen gelesen. Beim Bergabwandern und somit kurz vor dem Ende der Bergtour fand nur die Tafel am Treibholzrechen eine größere Aufmerksamkeit. Die Personen, die den Weg nur teilweise begingen, waren überwiegend Einheimische, die den Lehrpfad schon früher angeschaut hatten und daher weniger interessiert waren.

Die Unterschiede im Interesse für einzelne Inhalte können nicht nur anhand des Lehrpfades, sondern

\begin{tabular}{|l|c|c|c|c|}
\hline & $\begin{array}{c}\text { Pfad teilweise } \\
\text { begangen }\end{array}$ & $\begin{array}{c}\text { bergauf (und bergab) } \\
\text { begangen }\end{array}$ & $\begin{array}{c}\text { nur bergab } \\
\text { begangen }\end{array}$ & alle Passanten \\
\hline Treibholzrechen & $23 \%$ & $40 \%$ & $21 \%$ & $34 \%$ \\
Wasserwirtschaft & $14 \%$ & $34 \%$ & $5 \%$ & $24 \%$ \\
Lebensraum Tiere & $33 \%$ & $42 \%$ & $5 \%$ & $27 \%$ \\
Lebensraum Pflanzen & $13 \%$ & $41 \%$ & $0 \%$ & $35 \%$ \\
Verbauung & - & $23 \%$ & $5 \%$ & $17 \%$ \\
\hline
\end{tabular}

Tab. 2: Wahrnehmung der Lehrpfadtafeln abhängig von der zurückgelegten Wegstrecke 


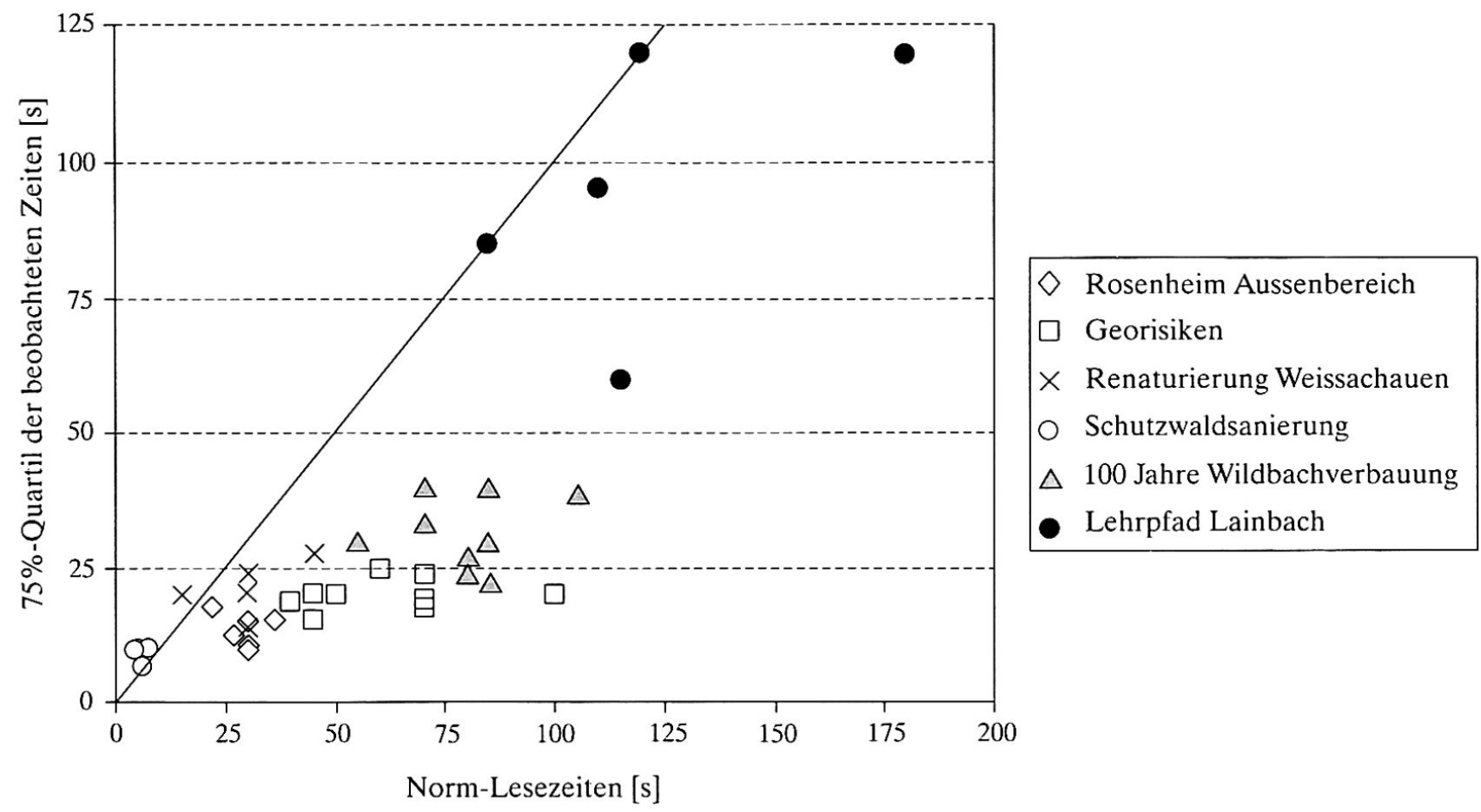

Abb. 1: Vergleich des 75\%-Quartils der Betrachtungszeiten mit den Norm-Lesezeiten der beobachteten Schautafeln. Auf der durchgezogenen Linie ist das 75\%-Quartil gleich der Norm-Lesezeit.

Comparison between the $75 \%$-quartil of the observation time and the norm-reading time of the observed panels. On the solid line the $75 \%$-quartil equals the norm-reading time.

Comparaison entre le quartile $75 \%$ des temps d'observation et les temps de lecture normatifs des tableaux d'information observés. Sur la ligne continue, le quartile $75 \%$ est égal à la durée de lecture normalisée.

Entwurf: K. WAGNER, Graphik: L. BAUMANN

auch der Ausstellung in Rosenheim gezeigt werden. In der Ausstellung in Rosenheim betrachteten die meisten Besucher das Tunnelaquarium (81\% der Beobachteten). Circa 55\% der Besucher spazierten durch den Außenbereich und nur $20-37 \%$ beschäftigten sich mit den unterschiedlichen Ausstellungsteilen innerhalb des Gebäudes. Am längsten wurde dabei die Videovorführung betrachtet, durchschnittlich 2,5 Minuten (Median). An den einzelnen Ausstellungstafeln verweilten die Besucher deutlich kürzer (siehe Abb.1). Abbildung 1 vergleicht das 75\%-Quartil der Betrachtungszeit - Personen, die sich überdurchschnittlich viel Zeit für die Beschäftigung mit den Tafeln nahmen - mit der Norm-Lesezeit. Mit Ausnahme von sehr kurzen Norm-Lesezeiten liegen die beobachteten Zeiten deutlich unter den Norm-Lesezeiten, wobei die Werte innerhalb und zwischen den unterschiedlichen Tafelgruppen deutlich schwanken. Ausreißer nach oben sind trotz relativ langer Norm-Lesezeiten die Tafeln des Lehrpfads am Lainbach. Ausserdem wird mit Ausnahme dieser Lehrpfadtafeln der Trend offensichtlich, dass je textreicher eine Tafel ist, desto selektiver sie wahrgenommen wird.
Die Selektion von für den Betrachter interessanten Objekten war besonders beim Besuch der Ausstellung in Rosenheim unvermeidlich. Man bräuchte alleine 17,5 Minuten, um den Ausstellungsteil Georisiken vollständig zu lesen. Auf welche Themen die Besucher fokussierten, wird in den nächsten Kapiteln erläutert.

\subsection{Die «Naturbrille» der Betrachter}

Auf die offene Frage, welche zwei Botschaften aus der Ausstellung in Rosenheim mitgenommen wurden, beschäftigten sich die meisten Antworten allgemein mit Themen des Natur- und Umweltschutzes (76 Antworten) und nur in einem geringeren Umfang mit der Notwendigkeit und den Möglichkeiten des Schutzes vor Naturgefahren (61 Antworten). Die Intensität des Ausstellungsbesuchs hatte einen sehr geringen Einfluss auf das Antwortverhalten. Die Ausstellungsmacher wollten hauptsächlich den Schutzaspekt verdeutlichen, das Thema Ökologie der Gewässer aber nicht unberücksichtigt lassen. Dieses Konzept hat sich eher nicht bewährt, da die Besucher mit ihrer «Naturbrille» die Fische im Aquarium, einen schönen Wildbach, die gezähmte Natur betrachteten, ohne 
Verbauung allgemein

Ungewöhnliche technische Schutzmaßnahmen

Maßnahmen sinnvoll an großen Gewässern

Umsiedeln/Flächenvorsorge

\author{
Eigenvorsorge \\ Sinnvolle technische \\ Schutzmaßnahmen
}

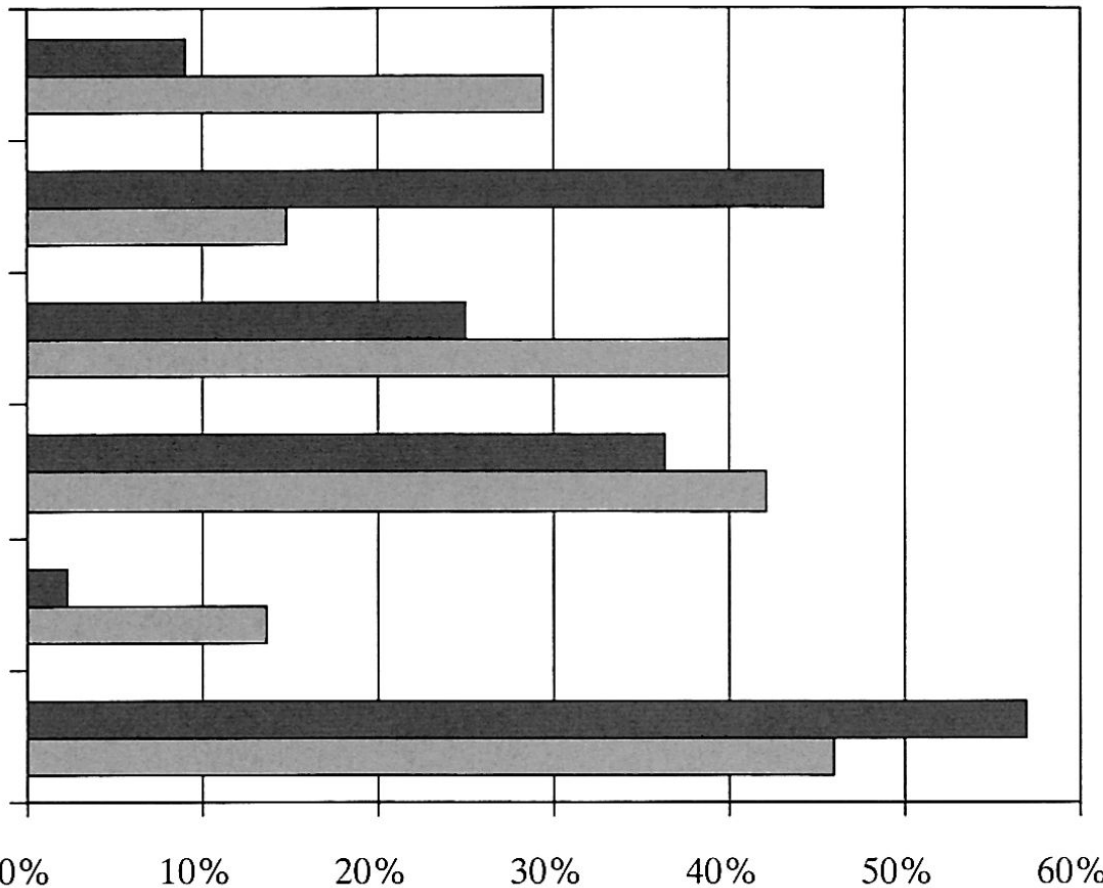

Anteil der Befragten

Ausstellung Rosenheim

Lehrpfad am Lainbach

Abb. 2: Vorgeschlagene Schutzmassnahmen für ein Dorf, das durch einen nicht verbauten Wildbach geschädigt wurde.

Proposed mitigation measures for a village damaged by an uncontrolled torrent.

Mesures de protection proposées pour un village qui a été endommagé par un torrent non aménagé.

verstärkt die Gefahren der ungezähmten Natur zu erkennen.

Auch beim Lehrpfad am Lainbach erinnerten sich die Befragten stärker an die Tafeln zum Naturraum als an die Tafeln, die sich allgemein mit der Wildbachverbauung bzw. den Aufgaben der Wasserwirtschaftsverwaltung beschäftigen. Einzige Ausnahme bildete die Tafel zum Treibholzrechen, die ja auch von einem größeren Anteil der Befragten wahrgenommen wurde.

\subsection{Die «Flachlandbrille»}

Von der Frage, welche Schutzmaßnahmen nach einem Hochwasser an einem unverbauten Wildbach ergriffen werden sollten, waren ein Drittel der Befragten in Rosenheim und ein Viertel der Befragten am Lainbach eher überfordert. Sie sprachen entweder allgemein davon, dass man den Bach verbauen müsste oder sie nannten nur eine der in Abb. 2 aufgeführten Maßnahmen. Ungefähr die Hälfte der Befragten nannte mindestens eine technische Schutzmaßnahme wie Sperren im Oberlauf bauen oder Treibholz entfernen. Meist spontan nach der Fragestellung empfahlen ca. 20\% der Befragten die Umsiedlung der betroffenen Häuser. Ebenfalls ca. $20 \%$ der Befragten forderten eine konsequente Flächenvorsorge. An die Möglichkeit der Eigenvorsorge dachten am Lainbach fast keine Befragten, in Rosenheim immerhin knapp 15\%.

Ein Drittel der Befragten beantwortete die Frage aus der Blickrichtung des Flachlands. Der Bau von Dämmen und die Schaffung von Retentionsflächen sind an großen Gewässern bekannte Maßnahmen, die die Befragten auf die Wildbäche übertrugen. Ebenso dürften die Vorschläge Umleiten des Baches und Schaffung von Wasserrückhaltebecken durch Analogieschlüsse spontan entwickelt worden sein. In der Wildbachverbauung werden sie dagegen nur äußerst selten eingesetzt und daher weder in der Ausstellung noch am Lehrpfad dargestellt.

In Rosenheim war der Anteil der Befragten, die von der Fragestellung überfordert waren bzw. «Flachlandlösungen» bevorzugten, deutlich größer. Zwei 
Erklärungsmöglichkeiten bieten sich für diesen Befund an: Am Lainbach waren zusätzlich zu den Erklärungen auf den Lehrpfadtafeln die Verbauungsmaßnahmen am Wildbach sichtbar. Der Befragte musste so nur die am Lainbach gesehenen Maßnahmen auf die Fragestellung übertragen. Zweitens waren $30 \%$ der Befragten am Lainbach Einheimische. Diese nannten deutlich mehr für Wildbäche typische Schutzmaßnahmen.

Obwohl die Ausstellungs- und Lehrpfadgestalter intensiv die technischen Schutzmaßnahmen gegen Wildbachgefahren verdeutlichten, bezog sich bei einem großen Teil der Befragten die Antwort auf ihr Alltags- und Medienwissen bzw. sie entwickelten spontan Lösungskonzepte. Die starke Betonung der Flächenvorsorge bzw. des Umsiedelns entspricht dabei den Botschaften, die den Medien entnommen werden, die nach Hochwasserereignissen an Rhein oder Elbe die Renaturierung der Gewässer und die Flächenvorsorge als beste Lösungskonzepte favorisieren.

\subsection{Wissens- und Einstellungsänderungen}

In den Befragungen wurde überwiegend deklaratorisches Wissen, d.h. Wissen, das unmittelbar der Ausstellung bzw. dem Lehrpfad entnommen werden konnte, abgefragt. Wie im letzten Kapitel dargestellt, beantworteten die Interviewpartner auch Fragen, bei denen das deklaratorische Wissen selbstständig auf einen neuen Bereich übertragen werden musste.

Veränderungen im deklaratorischen Wissen konnten beim Lehrpfad am Lainbach nur zum Teil nachgewiesen werden. Besucher, die erklärten, sie hätten den Lehrpfad am Befragungstag angeschaut, konnten die Fragen, die sich mit dem Treibholzrechen und den Gründen für seine Errichtung, den Aufgaben der Wasserwirtschaft und der Geologie beschäftigen, besser beantworten als die Nicht-Betrachter. Keine Unterschiede konnten bei diesen Gruppen für die Fragen nach Ökologie und Verbauungsmaßnahmen allgemein festgestellt werden. Zieht man nicht die Eigenaussage der Befragten, sondern die Beobachtungsergebnisse heran, ergibt sich ein ähnliches Bild. Die Betrachter der Tafel «Treibholzrechen» wussten hochsignifikant mehr als die Nicht-Betrachter. Für die Aufgaben der Wasserwirtschaftsverwaltung und die Tiere am Wildbach ist nur eine Tendenz in diese Richtung nachweisbar.

Für die im vorigen Kapitel erläuterte Frage nach den sinnvollen Schutzmaßnahmen für ein gefährdetes Dorf gab es am Lainbach keinen Unterschied zwischen den Betrachtern und Nicht-Betrachtern. In Rosenheim hatte nur die selbst eingeschätzte Intensität der Beschäftigung mit der Videovorführung einen signifikanten Einfluss auf die Anzahl der sachlich richtigen Antworten. Die Intensität der Beschäftigung mit dem Ausstellungsteil Wildbäche, der sich explizit mit dem
Thema auseinandersetzte, bzw. die Länge des Ausstellungsbesuchs hatten keinen Einfluss auf das Antwortverhalten. Antworten zu Einstellungsfragen wurden nicht durch den Ausstellungs- bzw. Lehrpfadbesuch beeinflusst.

\section{Diskussion}

Um die dargestellten Ergebnisse angemessen zu interpretieren, sollte berücksichtigt werden, dass das Naturgefahrenthema in der Regel einen sehr geringen Stellenwert im persönlichen und gesellschaftlichen Leben einnimmt (Geipel et al.1997:28; Sims \& Baumann 1983: 183). Also werden die angebotenen Informationen eher nicht wahrgenommen bzw. man beschäftigt sich nur oberflächlich damit, da das Thema nebensächlich ist. Diese Aussage soll durch die Beobachtungsergebnisse bei der Ausstellung in Rosenheim belegt werden. Die Hälfte der Besucher widmete sich den Inhalten der Ausstellung so wenig, dass sie keine vertieften Informationen über Naturgefahren aufnehmen konnte.

Die erste Frage, die nun interessiert, ist, welche Ausstellungsteile am ehesten die Aufmerksamkeit der Besucher auf sich ziehen konnten. Wie aus vielen anderen Ausstellungsevaluationen bekannt, ziehen vor allem belebte und außergewöhnliche Objekte die Besucher an (vgl. Scher 1998). Diese Anziehungskraft hat auch die Tafel am Treibholzrechen am Lainbach. Der Wanderer sieht zuerst das ungewöhnliche Bauwerk, das sich einer unmittelbaren Erklärung durch den Laien entzieht. Die Lehrpfadtafel wird so gerne und intensiv gelesen, da der Besucher sich eine unmittelbare Aufklärung über seine aktuellen Fragen wünscht. Die Position einer Informationstafel in der Landschaft sollte daher immer unmittelbar mit der Umgebung zusammenhängen.

Ein weiteres Selektionskriterium ist die «Naturbrille» der Betrachter. Informationen, die sich mit Natur (-schutz) beschäftigen, werden eher wahrgenommen bzw. besser erinnert. Die These von Deutsch (1973: 215), dass vermittelte Botschaften wie ein Schlüssel zu dem vorhandenen Wissensbestand, dem Schloss, passen sollten, wird mit dieser Untersuchung bestätigt.

Neben der Anziehungskraft einzelner Exponate ist nach Falk \& Dierking (1998: 68 ff.) auch deren Haltekraft (die Verweilzeit am Objekt) von Interesse. Wiederum weisen die belebten Objekte und die Videovorführung die höchsten Verweilzeiten auf. Bei den Ausstellungstafeln steigt zwar mit zunehmender Informationsfülle die Verweilzeit vor der einzelnen Tafel, die Informationsaufnahme wird dabei aber immer selektiver und außerdem nimmt die Anzahl der Betrachter bei textreichen Tafeln ab. 
Auch der Gesamtkontext einer Ausstellung beeinflusst die Intensität der Beschäftigung mit einzelnen Exponaten. Je größer die Ausstellung ist, desto weniger Zeit verbringen die Besucher an einzelnen Exponaten (FAlK \& Dierking 1998: 56). Besonders deutlich wurde dieser Effekt bei den Tafeln des Lehrpfads am Lainbach. Die Wanderer betrachteten die Tafeln deutlich länger als die Tafeln, die in der Ausstellung gezeigt wurden. Die einzeln stehende Tafel in der Landschaft wird spontan ohne den Kontext des Lehrpfads wahrgenommen und somit nur entsprechend des Interesses für dieses Thema gelesen.

Vor dem geschilderten Hintergrund wird deutlich, warum nach dem Ausstellungs- und Lehrpfadbesuch nur vereinzelt Wissens- und keine Einstellungsänderungen gemessen werden konnten. Die Besucher beschäftigten sich teilweise nur oberflächlich mit den dargebotenen Informationen und suchten sich überwiegend Informationen aus, die wie solche zur Ökologie der Gewässer eher der Bestätigung des eigenen Wissens dienen als dem Wissenszuwachs (vgl. FALK \& Dierking 1998: 74 ff.). Eine Ausnahme stellt die Tafel «Treibholzrechen» des Lehrpfads dar, da nur durch diese Tafel das außergewöhnliche Bauwerk verstanden werden kann.

\section{Folgerungen}

Dass der Rezipient die angebotenen Informationen selektiv entsprechend seiner Erfahrungen und Einstellungen auswählt, ist in der Medienwirkungsforschung vielfach gezeigt worden (MERTEN 1994: $297 \mathrm{ff}$.). Damit die zentral zu vermittelnden Botschaften rezipiert werden, bedarf es einer zielgerichteten Konzeption durch den Ausstellungsersteller. So hätte in Rosenheim eine inhaltliche Gliederung der Räume in «Natürlicher Bach - Folgen für den Menschen, Naturgefahrenprozesse, technische Schutzmaßnahmen, planerische Schutzmaßnahmen und Eigenvorsorge» auch dem eiligen Besucher das Grundkonzept des Naturgefahrenschutzes näher gebracht. Die thematische Gliederung wie in geogene Gefahren oder Wildbäche vermittelte dagegen keine deutlichen Botschaften an den Besucher. Ohne ein klares Konzept wird der Besucher sich nur mit dem beschäftigen, das vor dem Hintergrund seiner Erfahrung anknüpfungsfähig ist, d.h. er wird durch seine «Flachlandbrille» die Renaturierungsmaßnahmen an zu starr verbauten Wildbächen und durch seine «Naturbrille» Informationen über die Ökologie der Wildbäche aufnehmen.

Der Ausstellungsmacher sollte aktiv auf diese Brillen eingehen. Wildbachverbauungen werden von einem Teil der Bevölkerung spontan als ökologische und ästhetische Störung empfunden. Im ersten Ausstellungsraum sollten daher zwei Teilräume eingebaut sein, die man durch eine Tür mit einem Bild eines unverbauten bzw. eines verbauten Wildbachs betreten kann. In den Räumen werden dann die Folgen eines Hochwassers demonstriert. Die Besucher werden also zu Beginn bei ihrem Naturbild «abgeholt» und unmittelbar mit den Folgen des Ideals der unberührten Natur konfrontiert. Die Bereitschaft, sich mit den Schutzmaßnahmen auseinanderzusetzen, dürfte nach einem solchen Ausstellungsbeginn deutlich erhöht sein.

\section{Fazit}

Die beiden untersuchten Informationsinstrumente verfolgen entsprechend ihres Namens ein «Informationskonzept", in dem gut aufbereitete Fachinformationen rein kognitiv verarbeitet werden sollen. Zielführender wäre ein «Erlebniskonzept», das aktiv mit den Brillen der Betrachter spielt, d.h. die Empörung über die durch die Wildbachverbauung verschandelte Natur aufnimmt und darauf aufbauend die Notwendigkeit der Verbauungen erklärt. Neben der Katastrophe kann nur das Erlebnis das Naturgefahrenthema aus seinem «Mauerblümchendasein» herausholen. Der Titel eines Films der Bayerischen Wasserwirtschaftsverwaltung geht genau in diese Richtung: «Wildbäche: Faszination und Gefahr».

In von Naturgefahren bedrohten Gemeinden bietet sich eine zweigleisige Strategie an. Mittels stummer Zeugen - z.B. Hochwassermarken, bei Lawinen und Wildbächen bieten sich auch Objekte an, die die Ausdehnung des Ablagerungs- bzw. Überschwemmungsbereichs verdeutlichen - und Informationstafeln an außergewöhnlichen Verbauungsmaßnahmen wird eine ständige Sensibilisierung der Anwohner und Touristen für das Naturgefahrenthema erreicht. Mittels Übungen der Feuerwehr bzw. des Katastrophenschutzes können zusätzlich Ereignisse geschaffen werden, an denen die Anwohner zum Nachdenken über ihre Vorsorgebemühungen angeregt werden.

\section{Dank}

Das Projekt wurde von der Wasserwirtschaftsverwaltung in Bayern finanziert.

\section{Literatur}

Becker, W. \& E. Kistler (1992): Optimierung von Verkehrsaufklärungsmaßnahmen. - = Forschungsberichte der Bundesanstalt für Straßenwesen, Nr. 252, Bergisch Gladbach: 1-264.

Deutsch, K. (1973): Politische Kybernetik: Modelle und Perspektiven. - 3. Auflage, Freiburg: Rombach.

DiekmanN, A. (2001): Empirische Sozialforschung: 
Grundlagen, Methoden, Anwendungen. - 7. Auflage, Reinbek bei Hamburg: Rowohlt.

Falk, J. \& L. Dierking (1998): The museum experience. - Washington D.C.: Whalesback.

FRIEDRICHS, J. (1985): Methoden empirischer Sozialforschung. - 13. Auflage, Opladen: Westdeutscher Verlag. GeIPEL, R., HäRTA, R. \& J. PoHL (1997): Risiken im Mittelrheinischen Becken. - = Deutsche IDNDR-Reihe, Nr. 4, Bonn: 1-42.

LäNDERARBEITSGEMEINSCHAFT HoCHWASSER (im Auftrag der Umweltministerkonferenz) (1995): Leitlinien für einen zukunftsweisenden Hochwasserschutz: Hochwasser - Ursachen und Konsequenzen. - Stuttgart.

Lindell, M. \& R. Perry (2000): Household adjustments to earthquake hazard: A review of research. In: Environment and Behavior 32: 461-501.

Merten, K. (1994): Wirkungen von Kommunikation. In: Merten, K., Schmidt, S. \& S. Weischenberg (Hrsg.): Die Wirklichkeit der Medien: Eine Einführung in die Kommunikationswissenschaft. - Opladen: Westdeutscher Verlag: 291-328.

Rohrmann, B. (2000): Die Evaluation von Maßnahmen zur Risiko-Kommunikation: methodische Prinzipien und 2 Fallstudien. - In: Scholz, R. (Hrsg.): Erfolgskontrolle von Umweltmaßnahmen. - Heidelberg: Springer Verlag: 197-217.

SCHER, A. (Hrsg.) (1998): (Umwelt-)Ausstellungen und ihre Wirkungen. - = Schriftenreihe des Staatlichen Museums für Naturkunde und Vorgeschichte 7, Oldenburg: 1-232.

Schnell, R., Hill, P. \& E. Esser (1995): Methoden der empirischen Sozialforschung. - 5. Auflage, München, Wien: Oldenbourg Verlag.

Sims, J.H. \& D.D. Baumann (1983): Educational Programs and Human Response to Natural Hazards. - In: Environment and Behaviour 15, 2: 165-189.

WeichSELGARTNER, J. (2001): Naturgefahren als soziale Konstruktion: Eine geographische Beobachtung der gesellschaftlichen Auseinandersetzung mit Naturrisiken. - Aachen: Shaker Verlag.

\section{Zusammenfassung: Wie sag ich's der Bevölkerung? Evaluation einer Ausstellung und eines Lehrpfads zum Thema «Alpine Naturgefahren»}

Innerhalb des Projekts «Naturgefahrenbewusstsein und -kommunikation im Bayerischen Alpenraum» wurde aufbauend auf dem trimodalen Modell von Merten (1994) die Wirkung unterschiedlicher Informationsinstrumente über alpine Naturgefahren analysiert. Mit Hilfe einer Beobachtung und Befragung von Besuchern einer Ausstellung und eines Lehrpfads konnten selektive Wahrnehmungsroutinen identifiziert werden. Informationen, die sich mit der Natur bzw. Naturschutz beschäftigen, werden verstärkt wahrgenommen («Naturbrille»). Die Informationen werden vor dem Hintergrund der medialen Berichterstattung über Hochwasserereignisse an großen Flüssen bewertet und verarbeitet («Flachlandbrille»). Die Kommunikatoren sollten diese «Brillen» berücksichtigen und stärker das Erlebnis als Schlüssel der Wissensvermittlung in den Vordergrund rücken.

\section{Summary: How to Tell to the Public? \\ Evaluation of an exhibition and a nature trail about natural hazards in the Alps}

Within the project «natural hazard perception and communication in the Bavarian Alps» the effects of different information tools treating alpine hazard were analyzed using the trimodal model of Merten (1994). Selective perception routines were identified through visitor observations und quantitative surveys. Information on nature or nature conservation appear to receive most attention («nature filter»). Information is accessed and processed against the background of mass media reports of flooding at big rivers («flat land filter»). It is recommended that communicators should take these «filters» into consideration for new information campaigns, paying tribute to effects of key aspects such as «adventure» and "common knowledge" on knowledge transfer.

\section{Résumé: Comment le dire au public? Evaluation d'une exposition et d'un sentier didactique à partir du thème des dangers naturels alpins}

Au fil du projet «Connaissance et communication dans la région alpine bavaroise», les effets de divers instruments d'information sur les dangers naturels alpins ont été analysés, partant du model trimodal de Merten (1994). A l'aide d'une observation et d'une interrogation des visiteurs d'une exposition et d'un sentier didactique, des schèmes de perception étaient identifiables. Les informations concernant la nature ont été perçues de manière grossie («lunettes de la nature»). Les informations sont évaluées et transformées en fonction des rapports des media sur les grandes crues des rivières («lunettes des plaines fluviales»). Les communicateurs devaient prendre ces «lunettes» en considération, tenant à ce que l'expérience soit la clé de la connaissance.

Dipl.-Forstwirt. Klaus Wagner, Technische Universität München, Lehrstuhl für Forstpolitik und Forstgeschichte, Am Hochanger 13, D-85354 Freising. e-mail:wagner@forst.tu-muenchen.de

\section{Manuskripteingang/received/manuscrit entré le 5.8.2004 \\ Annahme zum Druck/accepted for publication/accepté pour l'impression: 8.3 .2005}

